\title{
CERTAIN INEQUALITIES INVOLVING FRACTIONAL POWERS
}

\author{
A. W. KEMP \\ (Received 1 March 1989; revised 14 August 1991) \\ Communicated by C. Sutherland
}

\begin{abstract}
The note re-examines Brown's new inequalities involving polynomials and fractional powers. Shorter proofs are provided, and greater attention is given to the conditions for the inequalities to hold.
\end{abstract}

1991 Mathematics subject classification (Amer. Math. Soc.): 26 D 05, 26 D20.

\section{Introduction}

Brown (1988) has investigated a class of inequalities involving polynomials and fractional powers. These were suggested by his work on singular measures; his intention is to reformulate the inequalities as measure theoretic results.

Brown's inequalities are tight, and are appealingly simple to state; such inequalities have a useful rôle in many branches of mathematics. Because they do not seem to fit into the usual categories of inequalities they are worthy of further study, particularly regarding the regions over which they hold.

The most interesting of the inequalities appears in

Brown's Proposition 1. Suppose that $s, t \geq 1$ and that $s^{-1}+t^{-1}=$ $\ln 3 / \ln 2$. Then

(C) 1992 Australian Mathematical Society 0263-6115/92 \$A2.00+0.00 


$$
1+x+x^{2} \geq\left(1+x^{s}\right)^{1 / s}\left(1+x^{t}\right)^{1 / t},
$$

for all $0 \leq x \leq 1$, if and only if $3(s+t) \leq 8$.

Brown commented that he had been able to find significant shortcuts for his other propositions, but thought his proof of Proposition 1 "still rather long".

The alternative proof in this note is much shorter.

A special case of $1+x+x^{2} \geq\left(1+x^{s}\right)^{1 / s}\left(1+x^{t}\right)^{1 / t}$ is

$$
1+x+x^{2} \geq\left(1+x^{s}\right)^{2 / s}
$$

Brown states that (2) holds for $x>0, s=\log _{3} 4$. But $s^{-1} \ln \left(1+x^{s}\right)$ is monotonically decreasing in $s$; also equality is attained when $x=0$. The statement can therefore be widened to

$$
1+x+x^{2} \geq\left(1+x^{s}\right)^{2 / s} \quad \text { for } x \geq 0, s \geq \log _{3} 4 .
$$

Clearly the conditions for

$$
1+x+x^{2} \geq\left(1+x^{s}\right)^{1 / s}\left(1+x^{t}\right)^{1 / t}, \quad s, t, x \text { real, } s \geq 1, t \geq 1,
$$

to hold are more general than those in Brown's Proposition 1.

As Brown may have realized, (4) is invariant when $x$ is replaced by $x^{-1}$, and so holds for $x \geq 1$ whenever it holds for $0<x \leq 1$.

In Section 2 we find that (4) is valid not only over the arc $s^{-1}+t^{-1}=$ $\ln 3 / \ln 2, s+t \leq 8 / 3$, but more generally in the (overlapping) regions

$$
s^{-1}+t^{-1} \leq \ln 3 / \ln 2, \quad s \geq s_{1}, t \geq s_{1}, 0 \leq x,
$$

and

$$
s^{-1}+t^{-1} \leq 1.5, \quad s \geq 1, t \geq 1,0 \leq x,
$$

where $s_{1}=1.0246 \ldots$ is the (unique) solution of $s^{-1}+t^{-1}=\ln 3 / \ln 2$, $s+t=8 / 3, s<t$.

The inequality does not of course hold for $s^{-1}+t^{-1}>\ln 3 / \ln 2,0 \leq x \leq$ 1. The present writer has been unable to establish general conditions for its validity within the two small remaining regions $1.5<s^{-1}+t^{-1}<\ln 3 / \ln 2$, $1<s<s_{1}$, and $1.5<s^{-1}+t^{-1}<\ln 3 / \ln 2,1<t<s_{1}$. Within the first of these, it does not hold for $s=1.005, t=1.72, x=0.1$; on the other hand, numerical studies suggest that it hold for $s=1.02, t=1.8,0 \leq x$.

Brown's other inequalities are re-examined briefly. 


\section{Brown's Proposition 1}

We begin by proving that inequality (4) holds when $0 \leq x \leq 1,3(s+t) \leq 8$ and $s^{-1}+t^{-1}=\ln 3 / \ln 2$. The necessity of the condition $3(s+t) \leq 8$ when $s^{-1}+t^{-1}=\ln 3 / \ln 2$ is easily checked (see Brown (1988)), so Proposition 1 follows readily. Inequality (4) and these conditions are symmetric in $s$ and $t$, and therefore we need only consider $s \leq t$. Let $s=c-a, t=c+a$, and (7) $f(x ; c, a)=\ln \left(1+x+x^{2}\right)-(c-a)^{-1} \ln \left(1+x^{c-a}\right)-(c+a)^{-1} \ln \left(1+x^{c+a}\right)$.

Then we seek to prove that $f(x ; c, a) \geq 0$ when $0 \leq x \leq 1,0 \leq a<c-1 \leq$ $1 / 3,1 /(c-a)+1 /(c+a)=\ln 3 / \ln 2$.

Now

$$
d f(x ; c, a) / d x=G(x ; c, a) /\left[\left(1+x+x^{2}\right)\left(1+x^{c-a}\right)\left(1+x^{c+a}\right)\right]
$$

where

$$
G(x ; c, a)=1+2 x-2 x^{2 c-1}-x^{2 c}-\left(x^{c-a-1}+x^{c+a-1}\right)\left(1-x^{2}\right) .
$$

When $s+t=8 / 3$, that is, $c=4 / 3$,

$$
G(x ; 4 / 3, a)=\left(1-x^{2}\right)\left(1-x^{1 / 3}\right)^{2}\{p(x)+q(x)\},
$$

where

$$
\begin{aligned}
p(x) & =\left(-x^{2 / 3}+2 x-2 x^{5 / 3}+x^{2}\right) /\left\{\left(1-x^{2}\right)\left(1-x^{1 / 3}\right)^{2}\right\} \\
& =-x^{2 / 3} /\left(1+x^{2 / 3}+x^{4 / 3}\right),
\end{aligned}
$$

which decreases monotonically from 0 to $-1 / 3$ for $0 \leq x \leq 1$, and

$$
\begin{aligned}
q(x) & =\left(1-x^{1 / 3-a}-x^{1 / 3+a}+x^{2 / 3}\right) /\left(1-x^{1 / 3}\right)^{2} \\
& =1-\left(x^{-a / 2}-x^{a / 2}\right)^{2} /\left(x^{-1 / 6}-x^{1 / 6}\right)^{2} \\
& =1-\sinh ^{2}\{0.5 a \ln (x)\} / \sinh ^{2}\{(1 / 6) \ln (x)\},
\end{aligned}
$$

which decreases monotonically from 1 to $1-9 a^{2}$. Moreover $\left(1-x^{2}\right)$ $\times\left(1-x^{1 / 3}\right)^{2} \geq 0$ for $0 \leq x \leq 1$. So $G(x ; 4 / 3, a)$, and hence $d f(x ; 4 / 3, a) /$ $d x$, either has no zero or exactly one zero in $0 \leq x \leq 1$, depending on whether $p(1)+q(1)=2 / 3-9 a^{2}$ is greater or less than zero. Consequently, since $f(0 ; c, a)=0$ and $[d f(x ; c, a) / d x]_{x=0}=1, f(x ; 4 / 3, a)$ either increases monotonically over $0 \leq x \leq 1$, or at first increases and then decreases. For $f(x ; c, a)$ to be non-negative for $0 \leq x \leq 1$, we therefore also require $f(1 ; 4 / 3, a)=\ln 3-\left(s^{-1}+t^{-1}\right) \ln 2 \geq 0$.

Consider now $g(u ; k)=u \ln \left(1+x^{1 / u}+\cdots+x^{k / u}\right), 0 \leq x \leq 1$. This is a convex function of $u$; therefore $g(u ; k)+g(v ; l)$, conditional on $A u+B v=$ $C, B / A>0$, is a convex function of $u$. Hence, if $u_{2} \leq u \leq u_{1}$ and 


$$
\begin{aligned}
C= & A u+B v=A u_{1}+B v_{1}=A u_{2}+B v_{2}, \\
& g(u ; k)+g(v ; l) \leq \max \left[g\left(u_{2} ; k\right)+g\left(v_{2} ; l\right), g\left(u_{1} ; k\right)+g\left(v_{1} ; l\right)\right] .
\end{aligned}
$$

Thus, setting $u=1 / s, v=1 / t$,

$$
g(1 / s ; k)+g(1 / t ; l) \leq \max \left[g\left(1 / s_{1} ; k\right)+g\left(1 / t_{1} ; l\right), g\left(1 / s_{2} ; k\right)+g\left(1 / t_{2} ; l\right)\right]
$$

where $s_{1} \leq s \leq s_{2}, C=A / s+B / t=A / s_{1}+B / t_{1}=A / s_{2}+B / t_{2}$.

This result, with $A=B=\ln 2, C=\ln 3, k=l=1$, and where $s=s_{1}=$ $1.0246 \ldots, t=t_{1}=1.6420 \ldots$, and $s=s_{2}=1.6420 \ldots, t=t_{2}=1.0246 \ldots$. are the solutions of the equations $s^{-1}+t^{-1}=\ln 3 / \ln 2$ and $s+t=8 / 3$, implies that inequality (4) is holds over the arc

$$
s^{-1}+t^{-1}=\ln 3 / \ln 2, \quad s+t \leq 8 / 3, \quad \text { for } 0 \leq x .
$$

Proposition 1 follows after checking the necessity of the condition $3(s+t) \leq 8$ when $s^{-1}+t^{-1}=\ln 3 / \ln 2$ (see Brown (1988)). (This argument avoids the appeal to Brown and Shepp's (1988) result).

REMARK 1. As noted in the introduction, (4) is invariant when $x$ is replaced by $x^{-1}$; so if it is valid for $0 \leq x \leq 1$, the inequality is valid for all non-negative $x$.

REMARK 2. Because $g(u ; 1)$ is an increasing function of $u$ when $0 \leq$ $x \leq 1, g(1 / s ; 1)$ is a decreasing function of $s$. Consequently, if (4) holds for $s=s_{3}, t=t_{3}$, it also holds for $s=s_{3}+\varepsilon, t=t_{3}+\delta$, where $\varepsilon \geq 0$, $\delta \geq 0$. Inequality (4) therefore holds over the region

$$
s^{-1}+t^{-1} \leq \ln 3 / \ln 2, \quad s \geq s_{1}, t \geq s_{1}, 0 \leq x,
$$

where $s_{1}=1.0246 \ldots$.

REMARK 3. It is trivial to show that $G(x ; 1.5,0.5)=x(1-x)$. Inequality (4) is valid therefore for $s=1, t=2$, and for $s=2, t=1$. Therefore, by result (13) and the positivity of $d g(u ; 1) / d u,(4)$ holds when $s^{-1}+t^{-1}=$ $1.5,1 \leq s, 1 \leq t$, and, more generally, over the region

$$
s^{-1}+t^{-1} \leq 1.5, \quad s \geq 1, t \geq 1,0 \leq x .
$$

REMARK 4. Combining (4) with (3) gives

$$
1+x+x^{2} \geq\left(1+x^{s}\right)^{1 / s}\left(1+x^{t}\right)^{1 / t} \geq\left(1+x^{2 / w}\right)^{w}, \quad 0 \leq x, w=s^{-1}+t^{-1},
$$

subject to the conditions in Remarks 2 and 3 (the lower bound can also be obtained via Holder's inequality). This can be reformulated in terms of hyperbolic functions as

$$
2^{-w}(1+2 \cosh y) \geq \cosh ^{1 / s}(s y / 2) \cosh ^{1 / t}(t y / 2) \geq \cosh ^{w}(y / w),
$$


for all real $y, w, s, t$ as above. The arguments leading to the conditions on $s$ and $t$ can also be restated using hyperbolic functions.

\section{Brown's other propositions}

The proofs of the other propositions can be made more unified; also the conditions on $s$ and $t$ can be made less restrictive. These inequalities are not invariant when $x$ is replaced by $1 / x$.

Let

$$
\begin{aligned}
f(x)= & \ln \left(1+x+\cdots+x^{j}\right)-s^{-1} \ln \left(1+x^{s}+\cdots+x^{k s}\right)-E^{\prime}\left(1 \ldots x^{l t}\right) \\
& -t^{-1} \ln \left(1+x^{t}+\cdots+x^{l t}\right),
\end{aligned}
$$

and

(20) $d f(x) / d x=G(x) /\left\{\left(1+x+\cdots+x^{j}\right)\left(1+x^{s}+\cdots+x^{k s}\right)\left(1+x^{t}+\cdots+x^{l t}\right)\right\}$.

Then for Proposition $2, j=l=2, k=1,0 \leq x \leq 1, s, t \geq 1$. To prove that $f(x) \geq 0$ when $s^{-1} \ln 2+t^{-1} \ln 3=\ln 3$, it suffices to show, with the aid of result (13), that $f(x) \rightarrow 0$ when $s \rightarrow \infty, t \rightarrow 1$, and that

$$
G(x) / x^{t-1}=2 x^{2-t}-1+x^{3-t}-2 x-2 x^{t}-4 x^{t+1}-2 x^{t+2}-x^{t+3}
$$

has at most one zero when $t=\ln 3 / \ln 1.5=2.7095 \ldots$ (compare with Brown (1988)). The former is self-evident, and the latter is true because $-2(t-2)<$ $-(3-t)$ and $x^{1-t}>x^{2-t}$ for $0 \leq x \leq 1$, and therefore

$$
d\left\{G(x) / t^{t-1}\right\} / d x=-2(t-2) x^{1-t}+(3-t) x^{2-t}-\cdots
$$

is always negative for $0 \leq x \leq 1$ when $t=2.7095 \ldots$.

Propositions 3(i), (ii), (iii) also all assume $0 \leq x \leq 1, s, t \leq 1$. They can be restated as follows:

3(i) $j=3, k=1, l=3 ; f(x) \geq 0$ whenever $s^{-1} \ln 2+t^{-1} \ln 4=\ln 4$;

3(ii) $j=3, k=2, l=2 ; f(x) \geq 0$ whenever $s^{-1} \ln 3+t^{-1} \ln 3=\ln 4$;

3(iii) $j=3, k=2, l=3 ; f(x) \geq 0$ whenever $s^{-1} \ln 3+t^{-1} \ln 4=\ln 4$.

The proofs follow immediately upon application of result (13) if we can show: for 3(i) that $f(x) \rightarrow 0$ for $s \rightarrow \infty, t \rightarrow 1$ (true), and that $x^{1-t} G(x)$ has at most one change of sign for $s=1, t=2$; for 3(ii) that $x^{1-t} G(x)$ has at most one change of sign for $s=1, t=\ln 3 / \ln (4 / 3)=3.8188 \ldots$ (a similar result for $s=\ln 3 / \ln (4 / 3)=3.8188 \ldots, t=1$ follows by symmetry); and for 3(iii) that $f(x) \rightarrow 0$ for $s \rightarrow \infty, t \rightarrow 1$ (true), and that $x^{1-t} G(x)$ has at most one change of sign for $s=1, t=\ln 4 / \ln (4 / 3)=4.8188 \ldots$ (compare with Brown (1988)). 
The expressions for $x^{1-t} G(x)$ in ascending powers of $x$ for the stipulated values of $s$ and $t$ in the three cases are:

$$
\begin{aligned}
\text { 3(i) } \quad x^{1-t} G(x)= & 1+2 x-2 x^{3}-4 x^{4}-6 x^{5}-4 x^{6}-2 x^{7}-x^{8} ; \\
3\left(\text { ii) } \quad x^{1-t} G(x)=\right. & 3 x^{3-t}-1+2 x^{4-t}-2 x+x^{5-t}-3 x^{2}-2 x^{t}-4 x^{t+1} \\
& -6 x^{t+2}-3 x^{t+3}-2 x^{t+4}-x^{t+5} ; \\
3(\text { iii }) \quad x^{1-t} G(x)= & 3 x^{3-t}+2 x^{4-t}-1+x^{5-t}-2 x-R(x),
\end{aligned}
$$

where the coefficients of the terms in $R(x)$ are all negative. For $3(\mathrm{i}), x^{1-t} G(x)$ is a concave function of $x$, also $\left[x^{1-t} G(x)\right]_{x=0}=1,\left[x^{1-t} G(x)\right]_{x=1}=$ -16 ; therefore $x^{1-t} G(x)$ changes sign exactly once. For 3(ii), we have $-3(t-3)<-2(4-t),-2<-(5-t)$ when $t=3.8188 \ldots$, and therefore $d\left\{x^{1-t} G(x)\right\} / d x$ is negative for $0 \leq x \leq 1$. Similarly, for 3(iii) we have $-3(t-3)<-(5-t)$ when $t=4.8188 \ldots$, and so $d\left\{x^{1-t} G(x)\right\} / d x$ is negative for $0 \leq x \leq 1$. Hence in each of the three cases, $G(x)$ has at most one zero in $0 \leq x \leq 1$, and the rest of the proof is straightforward.

REMARK 5. The functions $g(1 / s ; k)$ and $g(1 / t ; l)$ are decreasing functions of $s$ and $t$ respectively. Therefore, just as the condition $A / s+B / t=C$ in Brown's Proposition 1 can be relaxed, so can the restrictions on $s$ and $t$ for his other propositions. We have: for Proposition $2, s^{-1} \ln 2 / \ln 3+t^{-1} \leq$ 1 ; for $3(\mathrm{i}), s^{-1} / 2+t^{-1} \leq 1$; for 3 (ii), $s^{-1}+t^{-1} \leq \ln 4 / \ln 3$; and for 3(iii), $s^{-1} \ln 3 / \ln 4+t^{-1} \leq 1$.

\section{References}

G. Brown (1988), 'Some inequalities that arise in measure theory', J. Austral. Math. Soc. (Series A) 45, 83-94.

G. Brown and L. Shepp (1989), 'A convolution inequality', in: Contributions to Probability and Statistics, Essays in honour of Ingram Olkin (Springer, New York), pp. 51-57.

Mathematical Institute

University of St. Andrews

North Haugh, St. Andrews, Fife KY169SS

Scotland 